\title{
pneumonia
}

Review

\section{The role of rapid diagnostic tests in managing adults with pneumonia in low-resource settings}

\author{
Stephen J Aston ${ }^{\mathrm{a}, \mathrm{b}}$ \\ ${ }^{a}$ Malawi Liverpool Wellcome Trust Clinical Research Facility, Blantyre, Malawi; 'biverpool School of Tropical Medicine, Liverpool, United Kingdom
}

Corresponding author: Dr Stephen Aston, Wellcome Trust Clinical PhD Training Fellow, Malawi Liverpool Wellcome Trust Clinical Research Facility, PO Box 30096, Chichiri, Blantyre 3, Malawi. Phone: +2659970 123 13. Email: saston@liverpool.ac.uk

Author contributions: SJA met authorship criteria. SJA agrees with the manuscript's results and conclusions. SJA contributed entirely to the drafting and writing of the paper.

Received Feb 12, 2014; Accepted May 15, 2014; Published Jul 11, 2014

Citation: Aston S. The role of rapid diagnostic tests in managing adults with pneumonia in low-resource settings. pneumonia 2014;5:8-17

\begin{abstract}
In well-resourced settings the systematic use of rapid diagnostics tests (e.g. pneumococcal urinary antigen test) that define the causal pathogen to direct therapy has not resulted in significantly improved outcomes in adults with pneumonia. The management of pneumonia in many low-resource settings is complicated by a substantial burden of tuberculosis and HIV-associated opportunistic infections, in addition to the usual spectrum of pathogens seen in well-resourced settings. Clinical features alone do not reliably distinguish between these different aetiologies and physicians often have to treat empirically. Given the limitations in diagnostic laboratory capability present in most low-resource settings, rapid and point-of-care diagnostic tests could become valuable tools to guide treatment decisions. Pneumococcal and Legionella urinary antigen tests are specific and moderately sensitive, but their utility in low-resource settings is uncertain. The Xpert MTB/RIF (Cepheid, USA) platform and rapid assays for urinary lipoarabinomannan can substantially speed up tuberculosis diagnosis; the current challenge is to translate this into earlier treatment and hopefully improve patient outcome. In HIV-infected patients, 1-3- $\beta$-D-glucan is a serum marker of Pneumocystis jirovecii infection with excellent sensitivity. Further studies are needed to assess the clinical utility and cost-effectiveness of these rapid diagnostic assays when they are incorporated into treatment algorithms.
\end{abstract}

Keywords: BinaxNOW Streptococcus pneumoniae antigen card, lipoarabinomannan, Xpert MTB/RIF, 1-3- $\beta$-D-glucan, pneumonia 


\section{Introduction}

In well-resourced settings, rapid diagnostic tests to define the causative pathogen in community-acquired pneumonia (CAP) have failed to contribute significantly to patient management or improve outcomes [1]. In these settings, the spectrum of potential pathogens is generally well defined and empirical treatment algorithms are devised to reliably provide adequate antimicrobial cover [2]. Concordance to guidelines that define empirical treatment on the basis of severity, rather than tailoring of therapy on the basis of aetiological diagnostic tests, is important in achieving optimal outcomes [1]. The situation in low-resource settings is different. Frequently, the spectrum of pathogens is not well-defined; where data do exist, pathogens like Mycobacterium tuberculosis feature prominently [3]. In areas of high HIV-prevalence, such as most of sub-Saharan Africa, the majority of adults hospitalised with pneumonia are HIV-positive and are often highly immunocompromised, thus potentially harbouring a much broader range of pathogens [4]. Given this different landscape of disease, a pathogen-directed approach, at least to the level of distinguishing bacterial pneumonia from tuberculosis (TB) and Pneumocystis jirovecii or other opportunistic pathogens, is required. However, physicians in low-resource settings often only have access to the most rudimentary of laboratory diagnostic services. Point-of-care or rapid diagnostic tests to define the causative pathogen may potentially have great utility in these settings. This review discusses recent advances in point-of-care and rapid diagnostic tests for respiratory pathogens and their potential utility in lowresource settings, focusing particularly on areas of high HIV prevalence.

\section{Pneumococcal pneumonia}

Streptococcus pneumoniae is the most commonly identified causative organism of CAP in most settings [2,3]. Classical microbiological techniques of establishing the diagnosis of pneumococcal pneumonia (e.g. sputum Gram-stain, sputum or blood culture) are highly specific, but insensitive and often slow [5]. The Alere BinaxNOW Streptococcus pneumoniae Antigen Card (BinaxNOW-SP; Alere, USA) is an immunochromatographic test for the presence of the pneumococcal C-polysaccharide coat protein in urine; the test can produce a result within 15 minutes of the specimen being obtained and requires minimal laboratory infrastructure or staff expertise to perform [6]. Accurate assessment of the diagnostic performance characteristics of BinaxNOW-SP has been hampered by the lack of a sensitive and specific reference standard test. A recent meta-analysis that used a latent class model approach to adjust for the variable and imperfect nature of the reference standard, estimated the sensitivity and specificity of BinaxNOW-SP for the diagnosis of S. pneumoniae infection in adults with CAP was $74 \%$ and $97 \%$, respectively [5]. In practice, when used in conjunction with classical microbiological methods, the assay gives an incremental improvement in detection of pneumococcal pneumonia, particularly for patients already treated with antibiotics [6]. The mean duration of BinaxNOW-SP positivity is up to 8 weeks, hence it cannot be used as a test of cure and should not be used in cases of recurrent pneumonia less than 4 months after pneumonia caused by S. pneumoniae [6].

BinaxNOW-SP has poor specificity - and hence limited utility - in children owing to high rates of asymptomatic nasal carriage of S. pneumoniae [7]. In many low-resource settings there are also high rates of nasopharyngeal colonisation in adults $[8,9]$. In a recent study of 98 healthy Thai women, S. pneumoniae nasopharyngeal colonisation (present in $25 \%$ of women) was not associated with positive BinaxNOW-SP [10]. In well-resourced settings, the sensitivity and specificity of BinaxNOW-SP are reportedly unaffected by HIV-status [11]. The combined effect of HIV-infection and pneumococcal nasopharyngeal colonisation - which is common in low-resource settings - on the specificity of the test has not yet been studied in detail.

Regardless of its performance characteristics, the practical value of a diagnostic test for pneumococcal pneumonia merits careful consideration (Table 1). Recommended empirical therapy always includes anti-pneumococcal coverage. A confirmed diagnosis may be desirable in order to justify narrowing the spectrum of therapy with the aim of reducing the risk of complications of broad spectrum antibiotics (e.g. Clostridium difficile) and decreasing the prevalence of community antibiotic resistance [5]. Conversely, the possibility of polymicrobial infection and the potential benefit of combination therapies in cases of severe pneumococcal pneumonia, support continued broad empirical treatment even when pneumococcal infection is confirmed $[1,12]$. In a recent randomised trial, narrowing the spectrum of antibiotic treatment on the basis of pneumococcal and Legionella urinary antigen tests in adults hospitalised with CAP, failed to demonstrate any significant clinical or economic benefit and moreover was associated with a higher risk of clinical relapse [1]. In settings where the majority of patients hospitalised with pneumonia have HIV, and typically advanced immunosuppression, polymicrobial infection with not only other typical CAP pathogens but $M$. tuberculosis and other opportunistic pathogens is common [13, 14]. In this context, clinicians should have a low threshold for additional investigation, even after pneumococcal pneumonia is confirmed, if patients fail to improve or show clinical features highly suggestive of alternative aetiologies.

\section{Legionella pneumonia}

In well-resourced settings, Legionnaires' disease is responsible for $1-5 \%$ of cases of CAP overall and a higher proportion of severe disease $[15,16]$. Greater than $90 \%$ of reported cases of Legionnaires' disease are caused by Legionella pneumophilia and amongst these over $80 \%$ 
Table 1 Characteristics of the Streptococcus pneumoniae urinary antigen test (BinaxNOW-SP; Alere, USA) for the diagnosis of pneumococcal pneumonia

\begin{tabular}{ll}
\hline Advantages & Disadvantages \\
\hline $\begin{array}{l}\text { Urine specimen is easy to obtain and no processing } \\
\text { needed }\end{array}$ & High cost \\
$\begin{array}{l}\text { Minimal laboratory infrastructure and operator training } \\
\text { required }\end{array}$ & No information on antimicrobial resistance \\
Rapid results ( $\leq 15$ minutes) & Insufficient sensitivity to exclude pneumococcal \\
High specificity & pneumonia \\
Incremental improvement in sensitivity when combined & Early narrowing of antibiotic treatment not of proven \\
with blood and sputum culture & \\
Remains positive after starting antibiotics & \\
Facilitates early targeting of antibiotic treatment & \\
\hline
\end{tabular}

are caused by serogroup 1 strains [15], although there is considerable geographical variation in the predominance of particular Legionella species and L. pneumophilia serotypes.

Microbiological diagnosis of Legionella pneumonia is difficult. Its fastidious growth requirements mean that sputum and particularly blood culture are insensitive. Until recently, serology was the commonest means of diagnosis with both acute and convalescent specimens normally required for confirmation $[17,18]$.

Approximately $80 \%$ of patients with L. pneumophilia serogroup 1 infection excrete bacterial antigen in urine $[17,18]$. There are several available radio- and enzymeimmunoassays that detect urinary Legionella antigens with similar sensitivity and specificity, approximately $80 \%$ and $99 \%$, respectively [17]. More recently, an immunochromatographic version of the assay, analogous to BinaxNOW-SP, has been developed (Alere Binax Legionella Urinary Antigen Card; Alere) with comparable performance characteristics [19]. All of these urinary antigen assays only reliably detect serogroup 1 strains; their utility is therefore dependent upon the relative local predominance of L. pneumophilia serogroup 1 infection.

The burden of Legionella amongst adults with pneumonia in most low-resource settings is uncertain. Available data from sub-Saharan Africa indicate a very low prevalence of disease [3]. Assuming a low prevalence, the widespread use of Legionella diagnostic tests is not an appropriate use of scant resource. Equally, empirical treatment for Legionella and other atypical pathogens for all patients with moderate and severe pneumonia, as is recommended in most well-resourced settings, has substantial resource implications. Rather than attempting individual patient diagnosis, sentinel surveillance programmes or intermittent aetiological studies using serological testing to estimate the local burden of disease are a more practical way of informing treatment guidelines in low-resource settings.

\section{Tuberculosis}

\subsection{Burden and challenges}

In low-resource settings, particularly in sub-Saharan Africa, TB is common amongst patients presenting with pneumonia [3]. In African post-mortem series, TB is the most prominent cause of death of HIV-positive adults with respiratory infection [13]. In these settings, TB diagnosis is still largely reliant on smear microscopy and chest radiography despite their limited diagnostic accuracy; in HIV-associated TB, approximately only a quarter of cases are smear-positive and up to a quarter have normal radiographs [20]. The consequent delayed diagnosis not only causes increased morbidity for the affected patients, but permits increased onward transmission to contacts. The ideal diagnostic test needs to be sufficiently simple, robust and rapid, that it may be performed at the point-of-care, and give immediate results that are of sufficient specificity to justify treatment, whilst having excellent sensitivity in both HIV-negative and -positive patients, including those with advanced immunosuppression [21]. The last decade has seen renewed momentum in the area of TB diagnostics with new assays being developed, assessed, and now being incorporated into clinical practice [22] (Table 2). The following section will describe the development and recommended use of the urinary lipoarabinomannan (LAM) and Xpert MTB/RIF (Cepheid, USA) assays. 
Table 2 Diagnostic tests for pulmonary tuberculosis: current, under evaluation and in development

\begin{tabular}{|c|c|c|}
\hline Test & Comments & Ref \\
\hline Chest radiography & $\begin{array}{l}\text { Used as adjunct to smear microscopy. No radiographic pattern diagnostic of } \\
\text { TB and substantial inter-observer variation. Appearances vary with immune } \\
\text { status in HIV-associated disease. }\end{array}$ & 23 \\
\hline Sputum smear microscopy & $\begin{array}{l}\text { Rapid and widely available; often sole diagnostic test. Sensitivity is poor, } \\
\text { particularly in HIV-associated TB; increased by sputum concentration and } \\
\text { fluorescence microscopy rather than ZN staining. }\end{array}$ & 23 \\
\hline Sputum culture & $\begin{array}{l}\text { Gold-standard diagnostic test; often restricted to reference centres in } \\
\text { resource-limited settings. Automated liquid culture systems (e.g. BACTEC } \\
\text { MGIT 960; Becton Dickinson, USA) are faster and more sensitive than solid } \\
\text { culture techniques. }\end{array}$ & 23 \\
\hline Xpert MTB/RIF (Cepheid, USA) & $\begin{array}{l}\text { Fully automated NAAT platform; allows rapid detection of MDRTB. More } \\
\text { sensitive than sputum smear microscopy, particularly in HIV-infection. High } \\
\text { setup and running costs; needs uninterrupted electrical supply. }\end{array}$ & 22 \\
\hline $\begin{array}{l}\text { Line probe assay } \\
\text { (e.g. GenoType MTBDRplus 2.0; } \\
\text { Hain Lifescience GmbH, Germany) }\end{array}$ & $\begin{array}{l}\text { NAAT mainly used for rapid identification of isoniazid mono-resistance and } \\
\text { MDRTB in culture isolates. Now adapted for use on clinical specimens; } \\
\text { comparable accuracy to Xpert MTB/RIF. }\end{array}$ & 24 \\
\hline $\begin{array}{l}\text { Loop-mediated isothermal } \\
\text { amplification }\end{array}$ & $\begin{array}{l}\text { Simplified, manual NAAT for use in basic laboratory settings. Currently in } \\
\text { large scale evaluation; estimated sensitivity is } 88 \% \text { overall and } 56 \% \text { in } \\
\text { sputum smear-negative TB. }\end{array}$ & 25 \\
\hline $\begin{array}{l}\text { Urinary lipoarabinomannan } \\
\text { (e.g. Alere Determine TB LAM Ag; } \\
\text { Alere, USA) }\end{array}$ & $\begin{array}{l}\text { POC assay facilitating rapid treatment initiation. Most sensitive in advanced } \\
\text { HIV-infection; incremental increase in sensitivity over sputum smear } \\
\text { microscopy and Xpert MTB/RIF alone. }\end{array}$ & 26,27 \\
\hline Volatile organic compounds & $\begin{array}{l}\text { Mass spectrographic analysis of exhaled breath for compounds associated } \\
\text { with pulmonary TB. In early stages of development; requires sophisticated } \\
\text { instrumentation. }\end{array}$ & 21 \\
\hline Serological tests & $\begin{array}{l}\text { Humoral response to TB is highly variable; no single antibody measurement } \\
\text { proved useful in diagnosis. Assays based on simultaneous detection of } \\
\text { multiple antibodies in development. }\end{array}$ & 21 \\
\hline Proteomics & $\begin{array}{l}\text { Serum proteomic profile of active TB derived by mass spectrometry. } \\
\text { Diagnostic tests based on candidate biomarkers in development. }\end{array}$ & 21 \\
\hline
\end{tabular}

TB, tuberculosis; ZN, Ziehl-Neelsen; NAAT, nucleic-acid amplification test; MDRTB, multi-drug resistant tuberculosis; POC, point-ofcare.

\subsection{Diagnostic assays}

\subsubsection{Urinary lipoarabinomannan (LAM)}

Quite apart from the insensitivity of smear microscopy, sputum specimens are frequently difficult to obtain; many patients hospitalised with pneumonia are unable to produce a specimen of adequate quality. Sputum induction or other invasive means of obtaining lower respiratory tract specimens are often unavailable in low-resource settings or contraindicated due to patient instability. Diagnostic assays based on urine are potentially advantageous for several reasons: collection is straightforward - in even the sickest of patients - and does not generate potentially hazardous aerosols; specimens are safe to handle and of consistent quality [28].

The mycobacterial cell wall glycopeptide, LAM, is one of several mycobacterial antigens detectable in urine during TB infection [26]. LAM is released in large quantities from metabolically active or degrading cells and enters the systemic circulation [29] from disease sites or during mycobacteraemia and is subsequently filtered at the glomerulus to enter the urine $[26,30,31]$.

The first proof-of-principle assay for the detection of urinary LAM was developed by Hamasur et al [32]; urine specimens required extensive processing before being used in a 96 well-plate direct capture enzyme-linked immunosorbent assay (ELISA) format to detect LAM. Initial 
clinical evaluations demonstrated a sensitivity of $74 \%$ and specificity of $87 \%$ [33]. A similar assay developed by Chemogen Inc. (USA) for use with unprocessed urine showed similar sensitivity and improved specificity in a study of Tanzanian patients with suspected TB [34]. Further commercial versions of the assay have been developed (MTB ELISA; Chemogen Inc. and Clearview TB ELISA; Alere) and extensively evaluated in studies in Africa and Asia and a variety of clinical settings. The early promising results, however, have not been replicated; in meta-analyses, the pooled estimates of sensitivity of MTB ELISA and Clearview TB ELISA is $45 \%$ and $40 \%$, respectively $[26,27]$. Reported specificities have also varied considerably, ranging from $88 \%$ to $100 \%$, possibly reflecting differences in the reference standard diagnostic test used or the occurrence of disseminated disease [26]. All recent studies indicate a clear difference in the sensitivity of urinary LAM assays according to HIV-status; incremental improvement in sensitivity ranges from 3\% to $53 \%$ in HIV-positive subgroups [27]. The sensitivity also increases with the degree of immunosuppression such that it is as high as $85 \%$ in patients with CD4 counts less than 50 cells/ $\mu \mathrm{L}$; this is considerably greater than that of sputum smear microscopy in this patient group $[26,27]$.

The latest iteration of the LAM assay (Alere Determine TB LAM Ag; Alere) is a simplified lateral flow version [26]. This genuinely point-of-care test uses a small volume of unprocessed urine, gives results in around 30 minutes and has comparable performance characteristics to Clearview TB ELISA [35]. The test kits are relatively cheap, may be stored at room temperature and require minimal training to use [26]. Two large scale evaluations of the Alere Determine TB LAM Ag assay have helped to further define its role in TB diagnosis $[35,36]$. Not only is sensitivity greatest in patients with a low CD4 count ( $67 \%$ if CD4 $<50$ cells $/ \mu \mathrm{L}$ ), it is also significantly higher when other features of severe disease are present (i.e. advanced symptoms, haemoglobin $<8 \mathrm{~g} / \mathrm{dL}$, C-reactive protein [CRP] >200 $\mathrm{mg} / \mathrm{L}$ ] [20]. There is an incremental increase in sensitivity when urinary LAM assays and sputum smear microscopy are used in combination, indicating that these tests may detect different groups of TB patients [27, 35].

Urinary LAM assays are an important development in diagnostics for HIV-associated TB. Patients hospitalised with pneumonia in particular are a suitable target group for their use since they are likely to have the features of advanced immunosuppression and severe disease which are associated with the highest degree of assay sensitivity. In this group, a urinary LAM assay may function as useful rule-in test to facilitate rapid treatment initiation amongst some TB patients with the highest risk of mortality [37].

\subsubsection{Xpert MTB/RIF}

The development of the Xpert MTB/RIF platform is the single-most important recent development in TB diagnostics and has been extensively reviewed elsewhere [22]. This fully integrated and automated diagnostics platform is capable of identifying $M$. tuberculosis complex and the presence of mutations conferring rifampicin resistance in unprocessed sputum specimens in less than 2 hours [30]. World Health Organization (WHO) endorsement in 2010 and subsequent innovative funding strategies have permitted the widespread rollout of the Xpert MTB/RIF platform to many low- and middle-income countries and ensured its position as a central pillar of TB diagnostics in these settings [22].

The Xpert MTB/RIF has been extensively evaluated to determine its real-life diagnostic performance characteristics, operational feasibility and costeffectiveness. In a recent Cochrane systematic review including 18 studies that compared Xpert MTB/RIF against sputum culture, the overall pooled sensitivity was $88 \%$ and specificity was $98 \%$ [38]. The estimated sensitivity for sputum smear-positive and smear-negative subgroups was $98 \%$ and $67 \%$, respectively [38]. In HIV-associated TB, the sensitivity of Xpert MTB/RIF is estimated at $84 \%$ and is consistently better than sputum smear microscopy with a median increment of $30 \%$ [22].

The implementation of TB diagnostic algorithms based on Xpert MTB/RIF in low-resource settings has presented considerable challenges. Cost, training needs and other logistical constraints have to date largely restricted the rollout of the Xpert MTB/RIF platform to central laboratories. In settings of poor infrastructure, this separation of the patient and test result may impede the timely initiation of treatment [22]. A recent study demonstrated, however, that the Xpert MTB/RIF system can be accurately administered by a trained nurse in primary health-care centres to facilitate improved rates of same-day diagnosis and treatment initiation [39]. Future efforts will be focused on further rollout and decentralisation of the Xpert MTB/RIF system.

\section{Pneumocystis jirovecii pneumonia}

P. jirovecii pneumonia (PCP) is one of the commonest HIV-related opportunistic infections in well-resourced settings and should be considered in all HIV-positive patients presenting with features of respiratory infection [40]. Historically, definitive diagnosis has relied on direct visualisation of Pneumocystis organisms in bronchoalveolar lavage fluid and transbronchial tissue biopsy specimens (Table 3). Bronchoscopy is not routinely available for clinical care in most low-resource settings and so the burden of disease is unclear and traditionally assumed to be low [40-42]. However, several research bronchoscopy studies from African centres have demonstrated a considerable burden of PCP amongst HIV-infected patients 
Table 3 Summary of diagnostic tests for Pneumocystis jirovecii pneumonia (PCP)

\begin{tabular}{|c|c|c|}
\hline Test & Comments & Ref \\
\hline \multicolumn{2}{|l|}{ Microscopy } & \multirow[t]{5}{*}{45,46} \\
\hline $\begin{array}{l}\text { Cell wall stains (e.g. Toluidine } \\
\text { Blue } O \text {, calcofluor white) }\end{array}$ & $\begin{array}{l}\text { Identification of cysts or trophozoites in BALF or lung biopsy specimens; } \\
\text { 'gold-standard' diagnostic method. }\end{array}$ & \\
\hline \multirow{2}{*}{$\begin{array}{l}\text { Trophozoite stains (e.g. Grocott's } \\
\text { methenamine silver, Diff-Quick) }\end{array}$} & Immunofluorescence more sensitive than cytochemical stains. & \\
\hline & Only suitable for use with bronchoscopic or induced-sputum specimens. & \\
\hline Immunofluorescence & Training needed for accurate and consistent reporting. & \\
\hline \multicolumn{2}{|l|}{ Molecular assays } & \multirow[t]{5}{*}{$47-50$} \\
\hline PCR & Several genetic loci evaluated (e.g. mtLSUrRNA, HSP70, ITS, DHFR, MSG). & \\
\hline $\mathrm{nPCR}$ & High assay sensitivity may lead to false positive result if colonised with & \\
\hline \multirow[t]{2}{*}{ qPCR } & Pneumocystis; new quantitative assays have improved specificity. & \\
\hline & $\begin{array}{l}\text { May be used with oral wash and upper respiratory tract specimens with } \\
\text { reasonable sensitivity. }\end{array}$ & \\
\hline \multicolumn{3}{|l|}{ Serum assays } \\
\hline 1-3- $\beta$-D-glucan & $\begin{array}{l}\text { Cell wall component of Pneumocystis; highly sensitive marker of PCP. } \\
\text { Other invasive fungal infections and some antibiotics may cause false } \\
\text { positive results. }\end{array}$ & 51,52 \\
\hline S-adenosylmethionine & $\begin{array}{l}\text { Essential metabolic intermediate; scavenged from host during active } \\
\text { Pneumocystis infection such that serum levels are depleted. Poor diag- } \\
\text { nostic capability. }\end{array}$ & 53 \\
\hline
\end{tabular}

BALF, bronchoalveolar lavage fluid; PCR, single-round polymerase chain reaction; nPCR, nested PCR; qPCR, quantitative (realtime) PCR; mtLSUrRNA, mitochondrial large subunit rRNA; HSP70, heat shock protein 70; ITS, internal transcribed spacers; DHFR, dehydrofolate reductase; MSG, multicopy major surface glycoprotein; PCP, Pneumocystis jirovecii pneumonia

with features of respiratory infection [43-46]. An effective point-of-care test for PCP remains some way off, but there has been some relevant progress that merits discussion. In recent years, molecular assays have begun to supersede direct visualisation techniques for the detection of $P$. jirovecii [50]. The improved sensitivity of molecular assays based on polymerase chain reaction (PCR) may permit the use of non-invasive specimens (where Pneumocystis organisms are present in lower concentrations) such as expectorated sputum [47], nasopharyngeal aspirates [52] and oral washes [49] for the diagnosis of PCP, thus removing the need for bronchoscopy. The laboratory infrastructure required for these molecular assays in their current format still, however, precludes their use in most low-resource settings. Recently recognised serum markers indicative of Pneumocystis infection are an alternative diagnostic strategy for PCP that may be more feasible in the short-term $[53,54]$.

$1-3-\beta-D-g l u c a n$ is a common component of the cell wall of most pathogenic fungi, including Pneumocystis, and is released into the systemic circulation and detectable in serum during infection [56]. Several retrospective studies and a single prospective study are consistent in indicating that serum $1-3-\beta-D-$ glucan has excellent (>95\%) sensitivity and reasonable specificity (84-86\%) for detecting PCP in patients with both HIV and other forms of immunocompromise [54,57]. This degree of sensitivity is sufficient to screen patients with a low or moderate pre-test probability of disease, such that a negative result could reasonably be used to exclude PCP [54]. There are many alternative causes of an elevated serum $1-3-\beta-D-$ glucan that are clearly relevant during acute illness in an immunocompromised patient, including other invasive fungal infections (e.g. histoplasmosis, candidiasis), use of $\beta$-lactam antimicrobials obtained from Pencillium species, Gram-negative endotoxinaemia and renal failure [58]. PCP also frequently co-exists with other pathogens such that $a$ positive diagnosis may not justify narrowing the spectrum of treatment or discontinuing investigation [45]. Nonetheless, a diagnostic test for PCP with the precision of 1-3- $\beta$-D-glucan would represent a marked improvement on the empiricism that is currently practised in most low-resource settings where other diagnostic modalities are generally unavailable. The use of 1-3- $\beta$-D-glucan or other serum markers of PCP infection should be further assessed, and if shown to be promising, attempts made to develop assay formats that are 


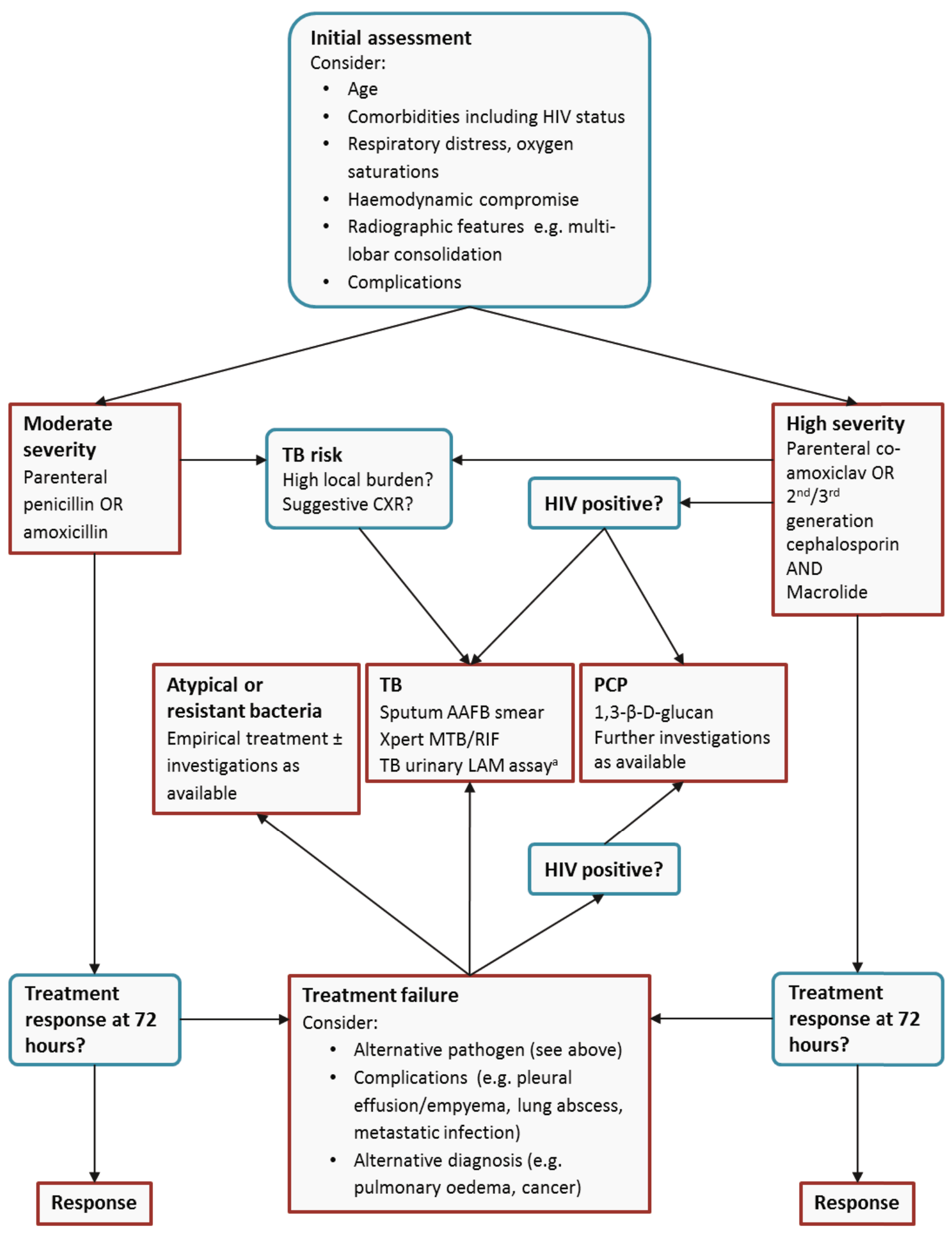

CXR, chest radiograph (x-ray); TB, tuberculosis; PCP, Pneumocystis jirovecii pneumonia; AAFB, acid fast bacilli; LAM, lipoarabinomannan

aHIV-positive patients only

Figure 1 An algorithm for the investigation of adults hospitalised with pneumonia in low-resource settings. Initial antimicrobial choice is determined following an assessment of disease severity. Initial investigations are selected on the basis of disease severity, HIV status, and the clinical likelihood of TB. Further investigations are performed in patients that fail to respond to initial treatment. This algorithm should be considered in conjunction with clinical features; empirical treatment for TB or PCP may be appropriate. 
adapted for use in low-resource settings [53].

\section{Considerations for use of rapid diagnostic assays}

The potential utility of point-of-care and rapid diagnostic tests for pneumonia in low-resource settings will relate to how frequently their results indicate a change in patient management and whether this change results in improved outcome. Recommendations for the use of diagnostic tests will vary between settings in line with the local prevalence of particular pathogens, the burden of HIV, and also the availability of alternative diagnostic tests. In the context of limited resources, diagnostic test use must be rationally targeted to specific patient groups such as those individuals with high severity disease or those that fail to improve with first-line treatment regimens.

A pneumonia management algorithm relevant to a low-resource setting that incorporates the use of rapid diagnostic tests is proposed in Figure 1. In this context, confirmation of pneumococcal aetiology by a urinary antigen test is unlikely to be a priority since the result will not substantially alter antimicrobial choice and it may not be an appropriate basis on which to curtail further diagnostic testing for TB. Urinary Legionella antigen tests are likely to be unaffordable in most low-resource settings; if used at all, they should be restricted to settings with a known burden of Legionellosis and for patients that fail to improve with first-line treatment. Assuming further positive evaluations, $1,3-\beta-D$-glucan may have a role in diagnosing PCP in the absence of other diagnostic tests.

\section{Conclusion}

In many low-resource settings the management of adults with pneumonia is complicated by a high incidence of TB and other HIV-associated opportunistic infections. This broad range of pathogens is encountered in settings where diagnostic microbiology laboratory capabilities are weak. The use of point-of-care and other rapid diagnostic tests that require minimal laboratory infrastructure to perform may provide a feasible adjunct to guide the management of pneumonia in low-resource settings. Further evaluation is needed to determine not only the diagnostic precision of rapid diagnostic tests in low-resource settings, but also whether management algorithms that incorporate and alter management on the basis of their results are associated with improved patient outcome.

Funding: This work was supported by the Wellcome Trust [Clinical PhD Grant 099962 to SJA]. The funder had no role in the design of this review, compilation and interpretation of material, decision to publish, or preparation of the manuscript.

Competing interests: SJA declares no competing interests.

Provenance and peer review: Commissioned; no funding has been requested or received by the authors for the preparation of the manuscript; externally peer reviewed.

Copyright: This is an open-access article distributed under the terms of the Creative Commons Attribution License, which permits unrestricted use, distribution, and reproduction in any medium, provided the original author and source are credited.

\section{References}

1. Falguera M, Ruiz-Gonzalez A, Schoenenberger JA, Touzon C, Gazquez I, Galindo C, et al. Prospective, randomised study to compare empirical treatment versus targeted treatment on the basis of the urine antigen results in hospitalised patients with community-acquired pneumonia. Thorax. 2010;65(2):101-6. http://dx.doi.org/10.1136/thx.2009.118588

2. Lim WS, Baudouin SV, George RC, Hill AT, Jamieson C, Le Jeune I, et al. BTS guidelines for the management of community acquired pneumonia in adults: update 2009. Thorax. 2009;64 Suppl 3:iii1-55. http://dx.doi.org/10.1136/ thx.2009.121434

3. Scott JA, Hall AJ, Muyodi C, Lowe B, Ross M, Chohan B, et al. Aetiology, outcome, and risk factors for mortality among adults with acute pneumonia in Kenya. Lancet. 2000;355(9211):1225-30. http://dx.doi.org/10.1016/S01406736(00)02089-4

4. SanJoaquin MA, Allain TJ, Molyneux ME, Benjamin L, Everett DB, Gadabu O, et al. Surveillance Programme of IN-patients and Epidemiology (SPINE): implementation of an electronic data collection tool within a large hospital in Malawi. PLoS Medicine. 2013;10(3):e1001400. http:// dx.doi.org/10.1371/journal.pmed.1001400

5. Sinclair A, Xie X, Teltscher $M$, Dendukuri N. Systematic review and meta-analysis of a urine-based pneumococcal antigen test for diagnosis of community-acquired pneumonia caused by Streptococcus pneumoniae. J Clin Microbiol. 2013;51(7):2303-10. http://dx.doi.org/10.1128/ JCM.00137-13

6. Kobashi Y, Yoshida K, Miyashita N, Niki Y, Matsushima T. Evaluating the use of a Streptococcus pneumoniae urinary antigen detection kit for the management of communityacquired pneumonia in Japan. Respiration. 2007;74(4):38793. http://dx.doi.org/10.1159/000092547

7. Adegbola RA, Obaro SK, Biney E, Greenwood BM. Evaluation of Binax now Streptococcus pneumoniae urinary antigen test in children in a community with a high carriage rate of pneumococcus. Pediatr Infect Dis. 2001;20(7):718-9. http://dx.doi.org/10.1097/00006454-200107000-00018

8. Hill PC, Akisanya A, Sankareh K, Cheung YB, Saaka M, Lahai G et al. Nasopharyngeal carriage of streptococcus pneumoniae in Gambian villagers. Clin Infect Dis 2006;43:673-679. http:// dx.doi.org/10.1086/506941

9. Adetifa IM, Antonio M, Okoromah CA, Ebruke C, Inem $\mathrm{V}$, Nsekpong $\mathrm{D}$, et al. pre-vaccination nasopharyngeal pneumococcal carriage ina Nigerian population:epidemiology and population biology. PLOS ONE 2012;7:e30458. http:// dx.doi.org/10.1371/journal.pone.0030548

10. Turner P, Turner C, Kaewcharernnet N, Mon NY, Goldblatt D, Nosten F. A prospective study of urinary pneumococcal antigen detection in healthy Karen mothers with high rates of pneumococcal nasopharyngeal carriage. BMC Infect Dis. 2011;11:108. http://dx.doi.org/10.1186/1471-2334-11-108 
11. Boulware DR, Daley CL, Merrifield C, Hopewell PC, Janoff EN. Rapid diagnosis of pneumococcal pneumonia among HIV-infected adults with urine antigen detection. J Infect. 2007;55(4):300-9. http://dx.doi.org/10.1016/j.jinf.2007.06.014

12. Waterer GW, Somes GW, Wunderink RG. Monotherapy may be suboptimal for severe bacteremic pneumococcal pneumonia. Arch Intern Med. 2001;161(15):1837-42. http://dx.doi.org/10.1001/archinte.161.15.1837

13. Bates M, Mudenda V, Mwaba P, Zumla A. Deaths due to respiratory tract infections in Africa: a review of autopsy studies. Curr Opin Pulm Med. 2013;19(3):229-37. http:// dx.doi.org/10.1097/MCP.0b013e32835f4fe4

14. Schleicher GK, Feldman C. Dual infection with Streptococcus pneumoniae and Mycobacterium tuberculosis in HIVseropositive patients with community acquired pneumonia. Intern J Tuberc Lung Dis : 2003;7(12):1207-8.

15. Diederen BM, Peeters MF. Evaluation of two new immunochromatographic assays (Rapid $U$ Legionella antigen test and SD Bioline Legionella antigen test) for detection of Legionella pneumophila serogroup 1 antigen in urine. J Clin Microbiol. 2006;44(8):2991-3. http://dx.doi. org/10.1128/JCM.00799-06

16. Helbig JH, Uldum SA, Bernander S, Luck PC, Wewalka $G$, Abraham B, et al. Clinical utility of urinary antigen detection for diagnosis of community-acquired, travelassociated, and nosocomial legionnaires' disease. J Clin Microbiol. 2003;41(2):838-40. http://dx.doi.org/10.1128/ JCM.41.2.838-840.2003

17. Den Boer JW, Yzerman EP. Diagnosis of Legionella infection in Legionnaires' disease. Eur J Clin Microbiol Infect Dis. 2004;23(12):871-8. http://dx.doi.org/10.1007/s10096-0041248-8

18. Waterer GW, Baselski VS, Wunderink RG. Legionella and community-acquired pneumonia: a review of current diagnostic tests from a clinician's viewpoint. Am J Med. 2001;110(1):41-8.http://dx.doi.org/10.1016/S00029343(00)00624-0

19. Helbig JH, Uldum SA, Luck PC, Harrison TG. Detection of Legionella pneumophila antigen in urine samples by the BinaxNOW immunochromatographic assay and comparison with both Binax Legionella Urinary Enzyme Immunoassay (EIA) and Biotest Legionella Urin Antigen EIA. J Med Microbiol. 2001;50(6):509-16.

20. Lawn SD, Kerkhoff AD, Vogt M, Wood R. HIV-associated tuberculosis: relationship between disease severity and the sensitivity of new sputum-based and urine-based diagnostic assays. BMC Med. 2013;11(1):231. http://dx.doi. org/10.1186/1741-7015-11-231

21. McNerney R, Daley P. Towards a point-of-care test for active tuberculosis: obstacles and opportunities. Nat Rev Microbiol. 2011;9(3):204-13. http://dx.doi.org/10.1038/nrmicro2521

22. Lawn SD, Mwaba P, Bates M, Piatek A, Alexander H, Marais $\mathrm{BJ}$, et al. Advances in tuberculosis diagnostics: the Xpert MTB/RIF assay and future prospects for a point-of-care test. Lancet Infect Dis. 2013;13(4):349-61. http://dx.doi. org/10.1016/S1473-3099(13)70008-2

23. Reid MJ, Shah NS. Approaches to tuberculosis screening and diagnosis in people with HIV in resource-limited settings. Lancet Infect Dis. 2009;9(3):173-84. http://dx.doi. org/10.1016/S1473-3099(09)70043-X

24. Crudu V, Stratan E, Romancenco E, Allerheiligen V, Hillemann A, Moraru N. First evaluation of an improved assay for molecular genetic detection of tuberculosis as well as rifampin and isoniazid resistances. J Clin Microbiol. 2012;50(4):1264-9. http://dx.doi.org/10.1128/JCM.05903-11

25. Mitarai S, Okumura $M$, Toyota E, Yoshiyama $T$, Aono $A$, Sejimo A, et al. Evaluation of a simple loop-mediated isothermal amplification test kit for the diagnosis of tuberculosis. Intern J Tuberc Lung Dis. 2011;15(9):1211-7, i. http://dx.doi.org/10.5588/ijtld.10.0629

26. Lawn SD. Point-of-care detection of lipoarabinomannan (LAM) in urine for diagnosis of HIV-associated tuberculosis: a state of the art review. BMC Infect Dis. 2012;12:103. http://dx.doi.org/10.1186/1471-2334-12-103

27. MinionJ, LeungE,TalbotE, DhedaK, PaiM,MenziesD.Diagnosing tuberculosis with urine lipoarabinomannan: systematic review and meta-analysis. Eur Respir J. 2011;38(6):1398-405. http:// dx.doi.org/10.1183/09031936.00025711

28. Peter J, Green C, Hoelscher M, Mwaba P, Zumla A, Dheda K. Urine for the diagnosis of tuberculosis: current approaches, clinical applicability, and new developments. Curr Opin Pulm Med. 2010;16(3):262-70. http://dx.doi.org/10.1097/ MCP.0b013e328337f23a

29. Sada E, Aguilar D, Torres M, Herrera T. Detection of lipoarabinomannan as a diagnostic test for tuberculosis. J Clin Microbiol. 1992;30(9):2415-8.

30. Lawn SD. Diagnosis of pulmonary tuberculosis. Curr Opin Pulm Med. 2013;19(3):280-8. http://dx.doi.org/10.1097/ MCP.0b013e32835f1b70

31. Talbot E, Munseri P, Teixeira P, Matee M, Bakari M, Lahey T, et al. Test characteristics of urinary lipoarabinomannan and predictors of mortality among hospitalized HIV-infected tuberculosis suspects in Tanzania. PloS one. 2012;7(3):e32876. http://dx.doi.org/10.1371/journal.pone.0032876

32. Hamasur B, Bruchfeld J, Haile M, Pawlowski A, Bjorvatn $B$, Kallenius $G$, et al. Rapid diagnosis of tuberculosis by detection of mycobacterial lipoarabinomannan in urine. J Microbiol Methods. 2001;45(1):41-52. http://dx.doi. org/10.1016/S0167-7012(01)00239-1

33. TessemaTA,HamasurB,BjunG,SvensonS, BjorvatnB.Diagnostic evaluation of urinary lipoarabinomannan at an Ethiopian tuberculosis centre. Scand J Infect Dis. 2001;33(4):279-84. http://dx.doi.org/10.1080/003655401300077306

34. Boehme C, Molokova E, Minja F, Geis S, Loscher T, Maboko $L$, et al. Detection of mycobacterial lipoarabinomannan with an antigen-capture ELISA in unprocessed urine of Tanzanian patients with suspected tuberculosis. Trans R Soc Trop Hyg. 2005;99(12):893-900. http://dx.doi.org/10.1016/j. trstmh.2005.04.014

35. Lawn SD, Kerkhoff AD, Vogt M, Wood R. Diagnostic accuracy of a low-cost, urine antigen, point-of-care screening assay for HIV-associated pulmonary tuberculosis before antiretroviral therapy: a descriptive study. Lancet Infect Dis. 2012;12(3):2019. http://dx.doi.org/10.1016/S1473-3099(11)70251-1

36. Peter JG, Theron G, van Zyl-Smit R, Haripersad A, Mottay L, Kraus $S$, et al. Diagnostic accuracy of a urine lipoarabinomannan strip-test for TB detection in HIVinfected hospitalised patients. Eur Respir J. 2012;40(5):121120. http://dx.doi.org/10.1183/09031936.00201711

37. Peter JG, Theron G, Dheda K. Can point-of-care urine LAM strip testing for tuberculosis add value to clinical decision making in hospitalised HIV-infected persons? PloS one. 2013;8(2):e54875. http://dx.doi.org/10.1371/journal.pone.0054875

38. Steingart KR, Sohn H, Schiller I, Kloda LA, Boehme CC, Pai M, 
et al. Xpert(R) MTB/RIF assay for pulmonary tuberculosis and rifampicin resistance in adults. The Cochrane Database Syst Rev. 2013;1:CD009593.

39. Theron G, Zijenah L, Chanda D, Clowes P, Rachow A, Lesosky $M$, et al. Feasibility, accuracy, and clinical effect of point-of-care Xpert MTB/RIF testing for tuberculosis in primary-care settings in Africa: a multicentre, randomised, controlled trial. Lancet. 2014;383(9915):424-35. http:// dx.doi.org/10.1016/S0140-6736(13)62073-5

40. Lowe DM, Rangaka MX, Gordon F, James CD, Miller RF. Pneumocystis jirovecii pneumonia in tropical and low and middle income countries: a systematic review and metaregression. PloS one. 2013;8(8):e69969. http://dx.doi. org/10.1371/journal.pone.0069969

41. Abouya YL, Beaumel A, Lucas S, Dago-Akribi A, Coulibaly $G$, N'Dhatz $M$, et al. Pneumocystis carinii pneumonia. An uncommon cause of death in African patients with acquired immunodeficiency syndrome. Am Rev Respir Dis. 1992;145(3):617-20. http://dx.doi.org/10.1164/ajrccm/145.3.617

42. Batungwanayo J, Taelman H, Lucas S, Bogaerts J, Alard $D$, Kagame $A$, et al. Pulmonary disease associated with the human immunodeficiency virus in Kigali, Rwanda. A fiberoptic bronchoscopic study of 111 cases of undetermined etiology. Am J Respir Crit Care Med. 1994;149(6):1591-6. http://dx.doi.org/10.1164/ajrccm.149.6.8004318

43. Aderaye G, Bruchfeld J, Aseffa G, Nigussie Y, Melaku K, Woldeamanuel $Y$, et al. Pneumocystis jiroveci pneumonia and other pulmonary infections in TB smear-negative HIV-positive patients with atypical chest X-ray in Ethiopia. Scand J Infect Dis. 2007;39(11-12):1045-53. http://dx.doi. org/10.1080/00365540701474508

44. Hargreaves NJ, Kadzakumanja O, Phiri S, Lee CH, Tang X, SalaniponiFM, etal. Pneumocystis cariniipneumonia in patients being registered for smear-negative pulmonary tuberculosis in Malawi. Trans R Soc Trop Med Hyg. 2001;95(4):402-8. http:// dx.doi.org/10.1016/S0035-9203(01)90197-X

45. Hartung TK, Chimbayo D, van Oosterhout JJ, Chikaonda T, van Doornum GJ, Claas EC, et al. Etiology of suspected pneumonia in adults admitted to a high-dependency unit in Blantyre, Malawi. Am J Trop Med Hyg. 2011;85(1):105-12. http://dx.doi.org/10.4269/ajtmh.2011.10-0640

46. Worodria W, Okot-Nwang M, Yoo SD, Aisu T. Causes of lower respiratory infection in HIV-infected Ugandan adults who are sputum AFB smear-negative. Intern J Tuberc Lung Dis. 2003;7(2):117-23.

47. Cruciani M, Marcati P, Malena M, Bosco O, Serpelloni G, Mengoli C. Meta-analysis of diagnostic procedures for Pneumocystis carinii pneumonia in HIV-1-infected patients. Eur Respir J. 2002;20(4):982-9. http://dx.doi.org/10.1183/0 9031936.02.01372002

48. Harris JR, Marston BJ, Sangrujee N, DuPlessis D, Park B. Costeffectiveness analysis of diagnostic options for pneumocystis pneumonia (PCP). PloS one. 2011;6(8):e23158. http:// dx.doi.org/10.1371/journal.pone.0023158

49. Larsen HH, Huang L, Kovacs JA, Crothers K, Silcott VA, Morris $A$, et al. A prospective, blinded study of quantitative touchdown polymerase chain reaction using oral-wash samples for diagnosis of Pneumocystis pneumonia in HIV-infected patients. J Infect Dis. 2004;189(9):1679-83. http://dx.doi. org/10.1086/383322

50. Durand-Joly I, Chabe M, Soula F, Delhaes L, Camus D, DeiCas E. Molecular diagnosis of Pneumocystis pneumonia. FEMS Immunol Med Microbiol. 2005;45(3):405-10. http:// dx.doi.org/10.1016/j.femsim.2005.06.006

51. Huggett JF, Taylor MS, Kocjan G, Evans HE, Morris-Jones S, Gant $V$, et al. Development and evaluation of a real-time PCR assay for detection of Pneumocystis jirovecii DNA in bronchoalveolar lavage fluid of HIV-infected patients. Thorax. 2008;63(2):1549. http://dx.doi.org/10.1136/thx.2007.081687

52. To KK, Wong SC, Xu T, Poon RW, Mok KY, Chan JF, et al. Use of nasopharyngeal aspirate for diagnosis of pneumocystis pneumonia. J Clin Microbiol. 2013;51(5):1570-4. http:// dx.doi.org/10.1128/JCM.03264-12

53. Sax PE, Komarow L, Finkelman MA, Grant PM, Andersen J, Scully E, et al. Blood (1->3)-beta-D-glucan as a diagnostic test for HIV-related Pneumocystis jirovecii pneumonia. Clin Infect Dis. 2011;53(2):197-202. http://dx.doi.org/10.1093/cid/cir335

54. Karageorgopoulos DE, Qu JM, Korbila IP, Zhu YG, Vasileiou VA, Falagas ME. Accuracy of beta-D-glucan for the diagnosis of Pneumocystis jirovecii pneumonia: a meta-analysis. Clin Microbiol Infect. 2013;19(1):39-49. http://dx.doi. org/10.1111/j.1469-0691.2011.03760.x

55. de Boer MG, Gelinck LB, van Zelst BD, van de Sande WW, Willems LN, van Dissel JT, et al. beta-D-glucan and $\mathrm{S}$-adenosylmethionine serum levels for the diagnosis of Pneumocystis pneumonia in HIV-negative patients: a prospective study. J Infect. 2011;62(1):93-100. http:// dx.doi.org/10.1016/j.jinf.2010.10.007

56. Desmet $S$, Van Wijngaerden E, Maertens J, Verhaegen J, Verbeken E, De Munter P, et al. Serum (1-3)-beta-D-glucan as a tool for diagnosis of Pneumocystis jirovecii pneumonia in patients with human immunodeficiency virus infection or hematological malignancy. J Clin Microbiol. 2009;47(12):38714. http://dx.doi.org/10.1128/JCM.01756-09

57. Onishi A, Sugiyama D, Kogata $Y$, Saegusa J, Sugimoto $T$, Kawano $S$, et al. Diagnostic accuracy of serum 1,3-betaD-glucan for pneumocystis jiroveci pneumonia, invasive candidiasis, and invasive aspergillosis: systematic review and meta-analysis. J Clin Microbiol. 2012;50(1):7-15. http:// dx.doi.org/10.1128/JCM.05267-11

58. Green MR. A modicum of caution for blood (1->3)-beta-Dglucan testing for Pneumocystis jurovecii in HIV-infected patients. Clin Infect Dis. 2011;53(10):1039-40; author reply 40. http://dx.doi.org/10.1093/cid/cir634 